Available online at GSC Online Press Directory

GSC Biological and Pharmaceutical Sciences

e-ISSN: 2581-3250, CODEN (USA): GBPSC2

Journal homepage: https://www.gsconlinepress.com/journals/gscbps

(RESEARCH ARTICLE)

\title{
Isolation and identification of the causative agent of bacterial throat infection according to a response to commonly antibiotic
}

\author{
Amany Eltayib Ataelmanan ${ }^{1}$, Adam Dawoud Abakar ${ }^{1}$ and Elsadig Mohammed Hamdan 2,* \\ ${ }^{1}$ Faculty of Medical Laboratory Sciences, University of Gezira, Wad Medani, Sudan. \\ ${ }^{2}$ A l-Gahd International Colleges for Applied Medical Sciences, KSA.
}

Publication history: Received on 16 July 2020; revised on 29 July 2020; accepted on 30 July 2020

Article DOI: https://doi.org/10.30574/gscbps.2020.12.1.0227

\begin{abstract}
Bacterial throat infection has a worldwide distribution, affecting persons of all ages, races, social strata and both genders. The present study was a prospective cross-sectional study aimed to isolate and identify the bacteria which cause throat infection and their antibiotic susceptibility in Madani - Sudan. Throat swabs were collected from 100 patients attended to Ear, Nose and Throat (ENT) Teaching Hospital. Isolation and identification of bacteria were made by directly inoculated onto blood agar, Gram stain and biochemical test. All isolated organisms were tested for their in vitro antimicrobial susceptibility against various antibiotics using the Kirby-Baur disk diffusion method. The major organism isolated was $S$. aureus (60\%), and the least organism isolated was S. pneumonia (6.7\%). The maximum antibiotic sensitivity of the isolates was against were erythromycin, ceftriaxone, cefuroxime, and ciprofloxacin. In This study, most the samples are negative for bacterial infections. S. aureus is the most common causes of throat infections and resists to antibiotics compared to other bacteria showed no resistance to all antibiotics.
\end{abstract}

Keywords: Sore throat; Throat swab; S. aureus; Erythromycin

\section{Introduction}

Infections of the throat have a tremendous impact on public health. Respiratory tract infection (RTI) is considered as one of the major public health problems and a leading cause of morbidity \& mortality in many developing countries [1]. A sore throat is a painful inflammation of the mucous membranes lining the pharynx. A sore throat can result from infection (bacterial or viral), allergy, inflammation, trauma, malignancy, airway obstruction, and other abnormal processes [2]. The primary causative agents of bacterial sore throat infections are Streptococcus pyogenes (most common), Streptococcus group C \& G, Corynebacterium diphtheria, Haemophilus influenza, Bordetella pertussis, Treponema vincentii, Leptotrichia buccalis [3]. A sore throat affects a person in many ways, and the symptoms vary from one individual to another. Accordingly, some describe the peculiar symptoms of the disorder as a burning sensation, while others feel a tickling or scratchy sensation in the throat. By and large, a sore throat affects the person as a general sore feeling that starts at the back of the oral cavity, gradually spreading out into the region of the middle throat; these symptoms are generally felt along with varying degrees of pain in individual cases. A sore throat can usually be seen more as a symptom of another illness and arises as a result of inflammation in the sensitive tissues of the throat. The body responds by increasing the rate of circulation of blood in the throat as soon as the initial inflammation or irritation has begun. Therefore, the swelling and the pain felt in the throat, and the subsequent reddening of the tissues is an actual reaction of the immune system [4]. Throat swab specimens should be obtained from the surface of both tonsils or tonsillar fossae and the posterior pharyngeal wall. Other areas of the oral pharynx and mouth are not acceptable sites, and these sites should not be touched with the swab before or after the appropriate areas have been sampled also, false-negative results may be obtained if the patient has received antibiotics shortly before or at the time the throat

\footnotetext{
${ }^{*}$ Corresponding author: Elsadig Mohammed Hamdan
} 
swab is obtained [5,6]. The study was aimed to isolate and identify the bacteria which caused throat infection and their antibiotic susceptibility in Madani - Sudan.

\section{Material and methods}

The study is a prospective cross-sectional study conducted during the period between November and December 2014. Throat swabs were collected from 100 patients attended to ENT hospitals at Madani -Gezira state. Each swab was obtained in good light and used the tongue depressor to depress the tongue, and under aseptic condition swab was collected from the inflamed area by a sterile cotton swab. Within two hours of collection, the swab was delivered to the laboratory. A throat swab was collected before antimicrobial treatment has been started.

The Swab was directly inoculated onto blood agar, incubated aerobically at $37^{\circ} \mathrm{C}$ for overnight. Colonial identification was studied according to morphology, size, colour, edges, hemolysis, on blood agar, side views of colonies and fermentation. Identification was based on Gram staining, isolated strains were identified by catalase production, coagulase test, inhibition of growth by bacitracin disc or optician disc, oxidase and urease.

All isolated organisms were tested for their in vitro antimicrobial susceptibility against various antibiotics, using the Kirby-Baur disk diffusion method according to the Clinical and Laboratory Standards Institute guidelines [7]. The antibiotics disc used were erythromycin, azithromycin penicillin, cefaclor, ciprofloxacin, cefuroxime, ceftriaxone and amoxiclav. Antibiotic sensitivity discs were placed on each plate and incubated at $37^{\circ} \mathrm{C}$ for 24 hours. The plates were examined for zones of inhibition around each of the antibiotic disc. These were measured and compared with an interpretive chart to determine the sensitive, intermediate and resistant strain.

\section{Results and discussion}

A total number of one hundred throat swabs specimens $(n=100)$ were collected from patients with presented to ENT hospitals at Madani -Gezira state with clinical findings suggestive of the throat infection before starting antibiotics therapy, show demographics characteristics of the study population according to sex on (table 1) and an according to age on (figure 1). Symptoms varied from patient to patient, and $95 \%$ had a sore throat (figure 2)

Among the 45 positive the primary organism isolated was $S$. aureus $27 / 45(60 \%)$ and the least organism isolated was S. pneumonia 3/45(7\%) (Figure 4).The maximum antibiotic sensitivity of the isolates was against erythromycin, ceftriaxone, cefuroxime, and ciprofloxacin $45 / 45(100 \%)$ followed by amoxiclav 40/45(88\%) (Figure 5, 6, 7, 8).In the present study, a total of 45/100 (45\%) of patients suffer from a bacterial throat infection and 55 (55\%) of patients were presented with clinical findings of throat infection with no bacterial pathogen was isolated.[8] also reported near frequencies (42\%).Staphylococcus aureus was the predominant isolate in the present study ( $60 \%$ ), That is with agreement to [8] who reported results of isolates mostly were coagulase-positive staphylococci (59\%) and lower results had been reported by [3] which was reported Staphylococcus aureus represent $9 \%$.Sensitivity tests revealed most of the bacterial pathogens of throat sensitive to cefuroxime (100\%), erythromycin (100\%), ciprofloxacin (100\%), ceftriaxone (100\%), amoxiclav (91.1\%). Staphylococcus aureus was highly sensitive to cefuroxime, erythromycin, ciprofloxacin and ceftriaxone; this result was an agreement [8].

Table 1 Demographics characteristics of the study population according to sex $(n=100)$ :

\begin{tabular}{lll}
\hline Sex & Frequency & Percent \\
\hline Male & 44 & 44.0 \\
Female & 56 & 56.0 \\
Total & 100 & 100.0 \\
\hline
\end{tabular}


Amany et al. / GSC Biological and Pharmaceutical Sciences, 2020, 12(01), 267-272

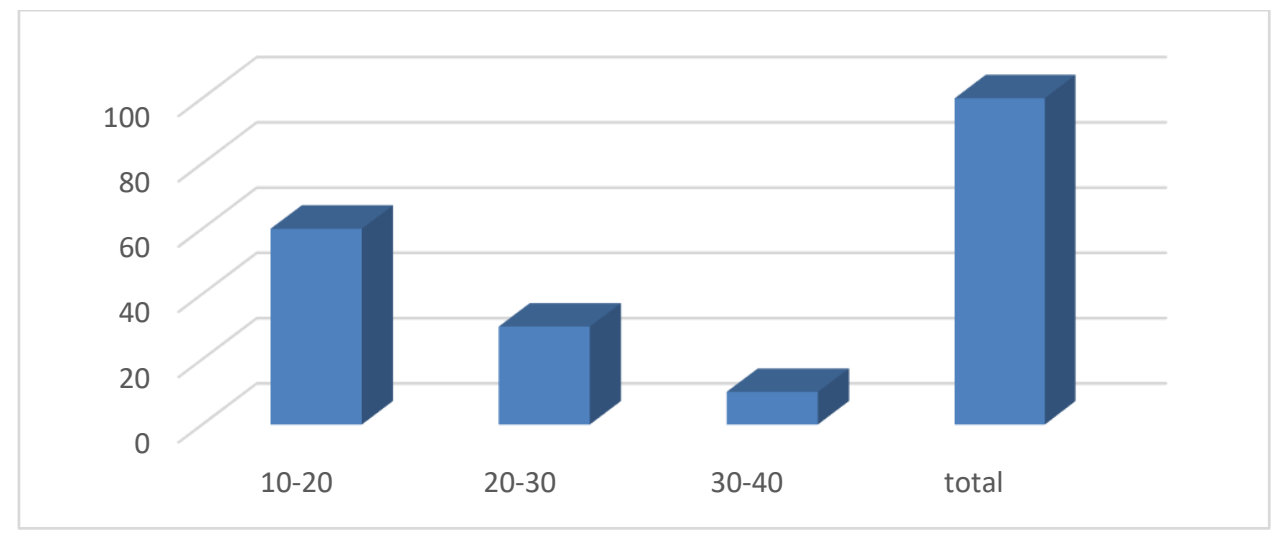

Figure 1 Frequency according to age group

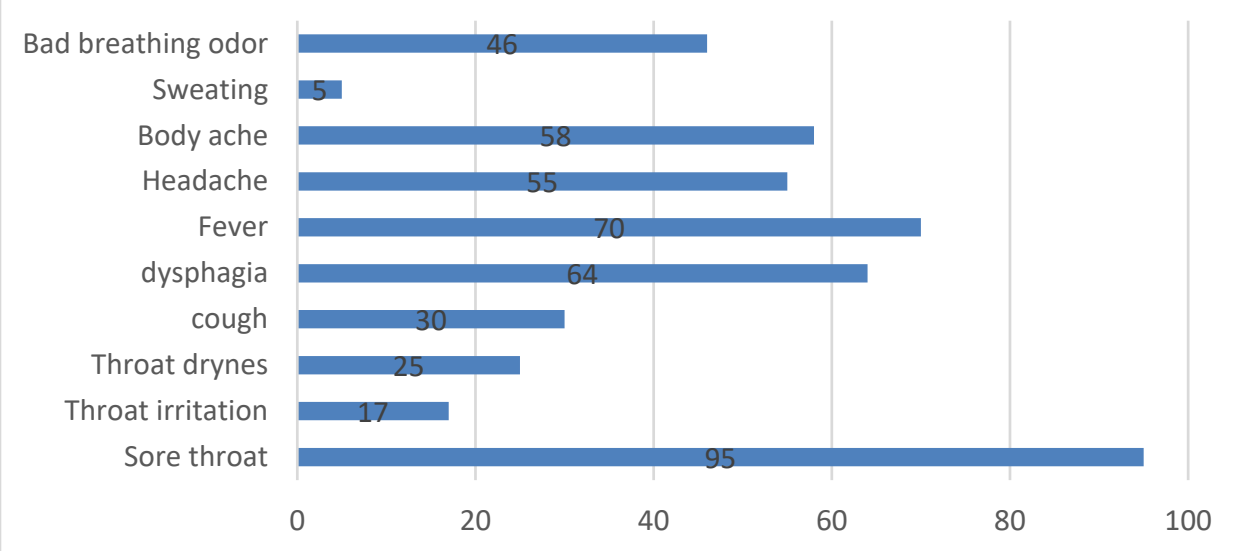

Figure 2 Distribution of symptoms in study group

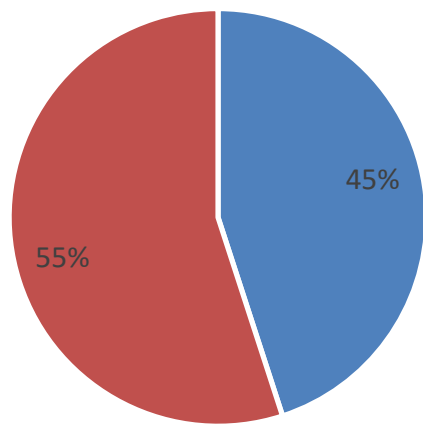

- bacteria - No pathogen organism isolated

Figure 3 Shown the percentage of isolates 


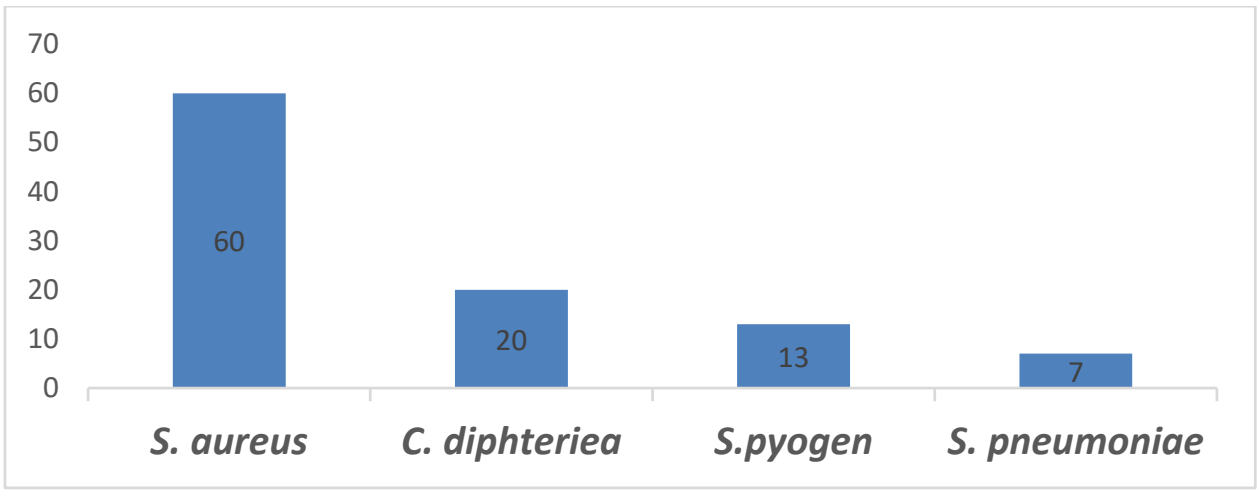

Figure 4 Frequency of isolates bacteria according to the species

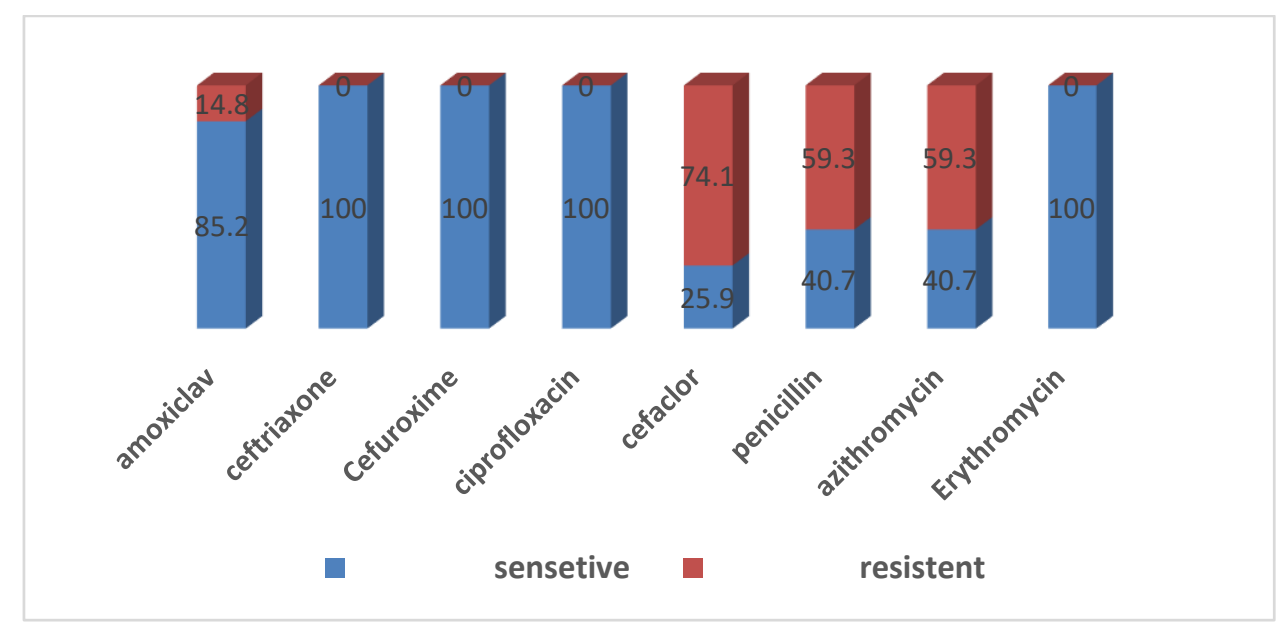

Figure 5 Sensitivity of $S$. aureus to antibiotics

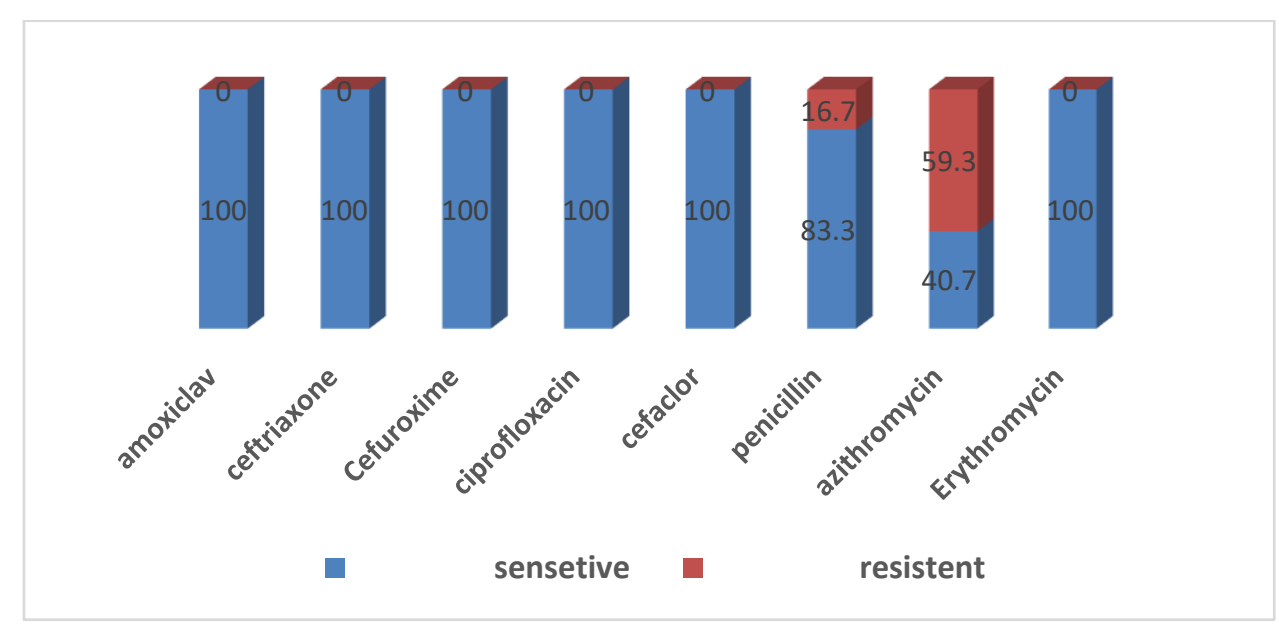

Figure 6 Sensitivity of S. pyogens to antibiotics 


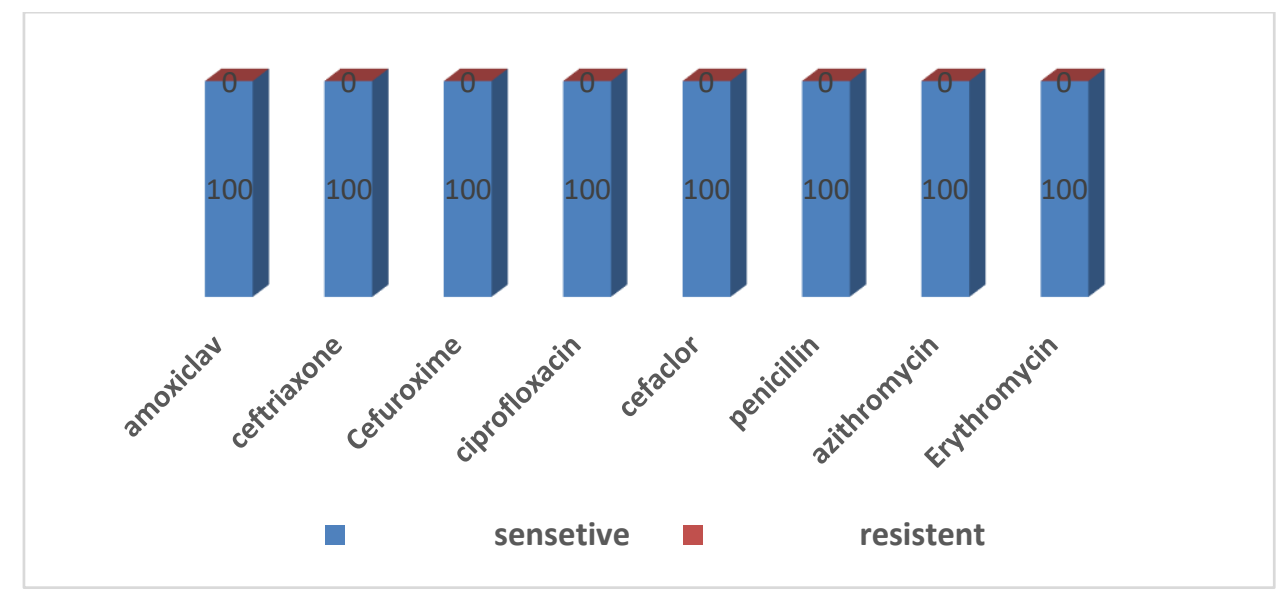

Figure 7 Sensitivity of $S$. pneumonia to antibiotics

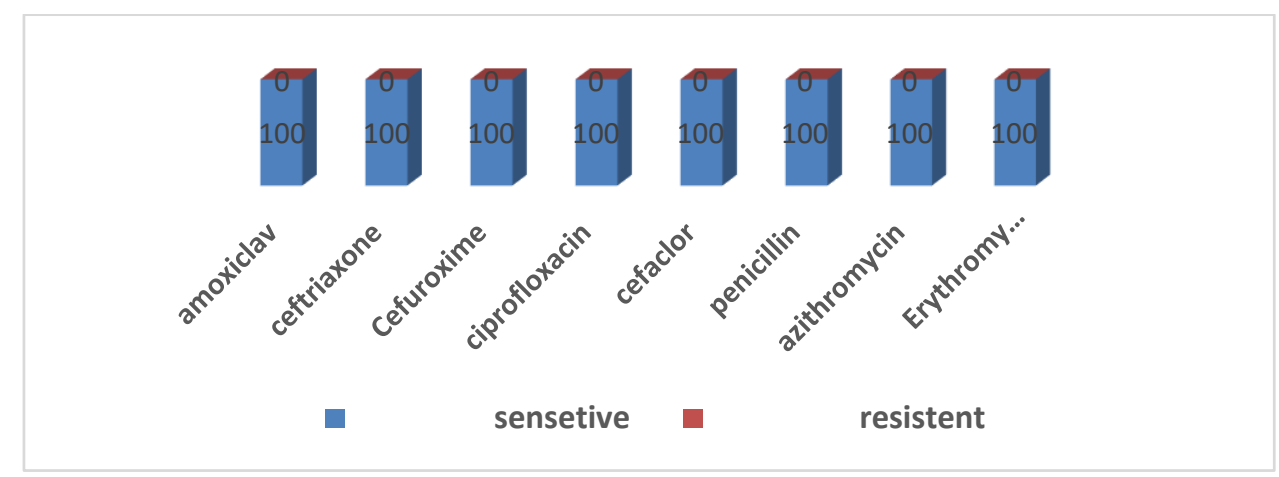

Figure 8 Sensitivity of Corynebacterium diphtheria to antibiotics

\section{Conclusion}

Throat infections can affect all age groups. S.aureus is the most common cause of throat infections and most bacteria that resistance to antibiotics. The most commonly used antibiotics (cefuroxime, ciprofloxacin, erythromycin, ceftriaxone) are sensitive by $100 \%$.

Throat swabs intended for URTI (upper respiratory tract infection) must be taken in routine especially in cases not responding to antibiotics, an antibiotics policy based on such studies should be formulated at the national loco-regional levels for effective use of antibiotics, Negative bacteria should be subjected for viral infection.

\section{Compliance with ethical standards}

\section{Acknowledgments}

We would like to extend our gratitude and appreciation to the Faculty of Medical Laboratory Sciences University of Gezira, and Professor Adam Dawoud Abakar Deportment of medical Parasitology College of Medical Laboratory Sciences University Gezira.

\section{Disclosure of conflict of interest}

The authors declare that there is no conflict of interest.

\section{Statement of informed consent}

Informed consent was obtained from all individual participants included in the study. 


\section{References}

[1] Yadav JK, Farooq U, Begum R, Tak V, Yadav SK, Kushwaha DKS and Pandey RK. (2015). Isolation and Identification of Organism from Throat Swab Along with Sensitivity Pattern from the Patients in North India. Annals of International Medical and Dental Research, 1(3), 2395-2814.

[2] Anitha M, Pratikshia K, Sulthan AM and Vijay M. (2016). Prevalence of bacterial growth in throat swab culture. International Journal of Pharmaceutical Science and Health Care, 6(2), 13-21.

[3] Somro A, Akram M, Khan MI, Asif HM, Sami A, Shah SAM and Rehman RU. (2011). Pharyngitis and sore throat: A review. African Journal of Biotechnology, 10(33), 6190-6197.

[4] Bisno AL, Gerber MA, Gwaltney Jr JM, Kaplan EL and Schwartz RH. (2002). Practice guidelines for the diagnosis and management of group A streptococcal pharyngitis. Clinical Infectious Diseases, 113-125.

[5] Van der Veen EL, Sanders EAM, Videler WJM, Van Staaij BK, Van Benthem PPG and Schilder AGM. (2006). Optimal site for throat culture: tonsillar surface versus posterior pharyngeal wall. European Archives of Oto-RhinoLaryngology and Head \& Neck, 263(8), 750-753.

[6] Reller LB, Weinstein M, Jorgensen JH and Ferraro MJ. (2009). Antimicrobial susceptibility testing: a review of general principles and contemporary practices. Clinical infectious diseases, 49(11), 1749-1755.

[7] Wakode PT, Gawarle SH, Joshi SV and Bajoriya R. (2003). Throat swab culture \& sensitivity reports-an overview. Indian Journal of Otolaryngology and Head and Neck Surgery, 55(2), 76-80.

[8] Moirangthem A and Gurung K. (2013). Bacteriological Analysis and Its Antibiogram Profile of Pharyngitis Cases from the Patients Attending Referral Hospital, Sikkim, India. Bali Medical Journal, 2(1), 10-13.

\section{How to cite this article}

Amany EA, Adam DA and Elsadig MH. (2020). Isolation and identification of the causative agent of bacterial throat infection according to a response to commonly antibiotic. GSC Biological and Pharmaceutical Sciences, 12(1), 267-272. 